\title{
Electronic Effects on the Reactivity of Cross-Conjugated Carbonyl Systems with $\mathrm{Fe}_{2}(\mathrm{CO})_{9}$
}

\author{
María C. Ortega-Alfaro ${ }^{*, a}$, José G. López-Cortés ${ }^{b}$, Rubén A. Toscano ${ }^{b}$ and Cecilio Alvarez-Toledano \\ ${ }^{a}$ Facultad de Química, Ed. A, Lab. 4-D, UNAM, Circuito Exterior, Ciudad Universitaria, \\ Coyoacán 04510, México D.F., México \\ ${ }^{b}$ Instituto de Química-UNAM, Circuito Exterior, Ciudad Universitaria, Coyoacán 04510, México D.F., México
}

\begin{abstract}
Neste trabalho foram estudados os efeitos eletrônicos induzidos por p-substituintes no anel fenilico de diversos compostos carbonílicos conjugados [4- $p$-R-fenil-1-(1,3-ditiolano-2-ilideno)3E-buteno-2-ona, onde $\left.\mathrm{R}=\mathrm{NO}_{2}, \mathrm{Cl}, \mathrm{Br}, \mathrm{OMe}, \mathrm{NEt}_{2}(2-6)\right]$, e sua reatividade frente a $\mathrm{Fe}_{2}(\mathrm{CO})_{9}$ usando o diagrama de Hammett. A estrutura dos compostos $(\mathbf{3}$ e $\mathbf{4 b})$ foi totalmente estabelecida por análise de difração de raio-X.
\end{abstract}

The electronic effects induced by $p$-substituents on the phenyl ring of several novel crossconjugated carbonyl compounds [4- $p$-R-phenyl-1-(1,3-dithiolane-2-ylidene)-3E-butene-2-one, where $\left.\mathrm{R}=\mathrm{NO}_{2}, \mathrm{Cl}, \mathrm{Br}, \mathrm{OMe}, \mathrm{NEt}_{2}(\mathbf{2}-\mathbf{6}),\right]$ and their reactivity towards $\mathrm{Fe}_{2}(\mathrm{CO})_{9}$ have been studied using a Hammett plot. The structure of two compounds ( $\mathbf{3}$ and $\mathbf{4 b}$ ) was fully established by X-ray diffraction analysis.

Keywords: iron(0) complexes, C-S bond activation, reactivity, Hammett plot, X-ray diffraction

\section{Introduction}

The $\alpha, \beta$-unsatured carbonyl compounds react with $\mathrm{Fe}_{2}(\mathrm{CO})_{9}$ forming $\eta^{2}-\left[\mathrm{Fe}(\mathrm{CO})_{4}\right]$ and the most thermodynamically stable $\eta^{4}$-[Fe(CO) $\left.{ }_{3}\right]$ complexes, ${ }^{1}$ which have shown different applications such as transfer agent of $\mathrm{Fe}(\mathrm{CO})_{3},{ }^{2}$ protecting group, ${ }^{3}$ stereodirector group, ${ }^{4}$ etc. Recently, we have described how reactivity changes drastically when heteroatoms (nitrogen and sulfur) in $\beta$-position are located on the system leading to novel an unexpected dinuclear complexes. ${ }^{5}$ In this subject, we have reported that the reactivity of cross-conjugated carbonyl systems $\beta$-positioned by sulfur atoms towards $\mathrm{Fe}_{2}(\mathrm{CO})_{9}$, principally produce partial and total $\mathrm{C}-\mathrm{S}$ bond activation in addition to the formation of $\eta^{4}$-[Fe( $\left.\left.\mathrm{CO}\right)_{3}\right]$ complexes (Scheme 1). ${ }^{6}$ As a part of our constant interest in the understanding of the coordination patterns of this kind of systems, we herein report the study of electronic effects induced by $p$-substituents on the phenyl ring on the mechanism of reaction of cross-conjugated carbonyls

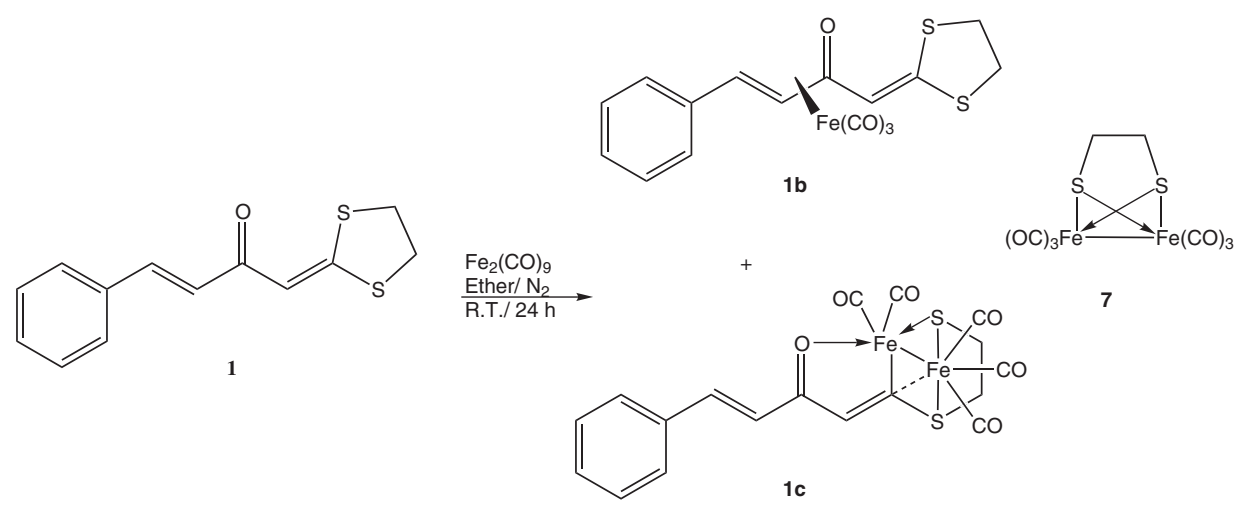

Scheme 1.

* e-mail: cecilio@servidor.unam.mx 


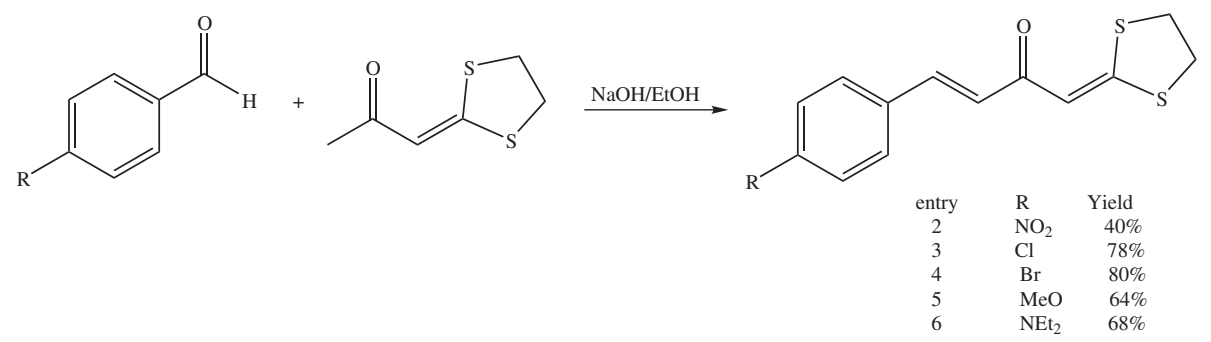

Scheme 2.

systems with $\mathrm{Fe}_{2}(\mathrm{CO})_{9}$, by correlating the electronic density on the $\mathrm{C}-3$ with the respective Hammett parameters.

\section{Results}

We have synthesized a series of ligands including electron-withdrawing and electron-donating groups on para-position of aromatic ring (Scheme 2). These ligands were totally characterized by the conventional spectroscopic techniques (MS, IR, ${ }^{1} \mathrm{H}$ and ${ }^{13} \mathrm{C} \mathrm{NMR}$ ) and they have some similarities in their ${ }^{1} \mathrm{H}$ and ${ }^{13} \mathrm{C}$ NMR spectra such as, a multiple signal around $3.4 \mathrm{ppm}$ that corresponds to the methylene groups and one simple signal around 6.8 ppm assigned to the $\underline{H C C S S}$ in ${ }^{1} \mathrm{H}$ NMR. In ${ }^{13} \mathrm{C}$ NMR, they exhibit two signals in 35 and 39 ppm for the methylene groups of the dithiolane system, the corresponding signal of HCCSS shifted at $112 \mathrm{ppm}$, the $\underline{C S S}$ around $165 \mathrm{ppm}$, and the CO group at $185 \mathrm{ppm}$. The IR spectra of these ligands show a weak narrow band around $1630 \mathrm{~cm}^{-1}$ assigned to $\mathrm{CO}$ group, a strong fine band in $1500 \mathrm{~cm}^{-1}$, attributed to $\mathrm{C}=\mathrm{C}$ bond, in addition to the respective bands for each ligand. The band intensities for the $\mathrm{CO}$ and $\mathrm{C}=\mathrm{C}$ groups indicate that an $\mathrm{S}$... O interaction through to $\mathrm{C}-\mathrm{C}$ double bond is present as reported elsewhere. ${ }^{7}$

The structural arrangement for $\mathbf{3}$ was fully established by a single-crystal X-ray diffraction analysis (Figure 1). The $\mathrm{PhCH}=\mathrm{CHCO}$ moiety presents a -trans conformation. The ylidenbutenone backbone of the ligand is quasi-planar with the phenyl ring tilted $18.6^{\circ}$ out of main plane. The bond distance $\mathrm{C} 1-\mathrm{C} 11$ in comparison with $\mathrm{C} 3-\mathrm{C} 4$ bond is longer $[0.069 \AA]$ (Table1) and the distance [S1---O1, $2.705 \AA]$ is shorter than the $\sum$ of Van der Waals radii. These features indicate that an interaction between the sulfur and oxygen atoms through the conjugated system is present, in agreement with the obtained results from IR technique and with described in literature. ${ }^{6,7}$ The 1,3dithiolane ring adopts a half-chair conformation with $C_{2}$ axe over $\mathrm{C} 11$. Additionally, this moiety presents disorder generating two conformers in 46/54 ratio, only the major contributors are shown in Figure 1.

Magnetic stirring of a solution of 2 and $\mathrm{Fe}_{2}(\mathrm{CO})_{9}$ in

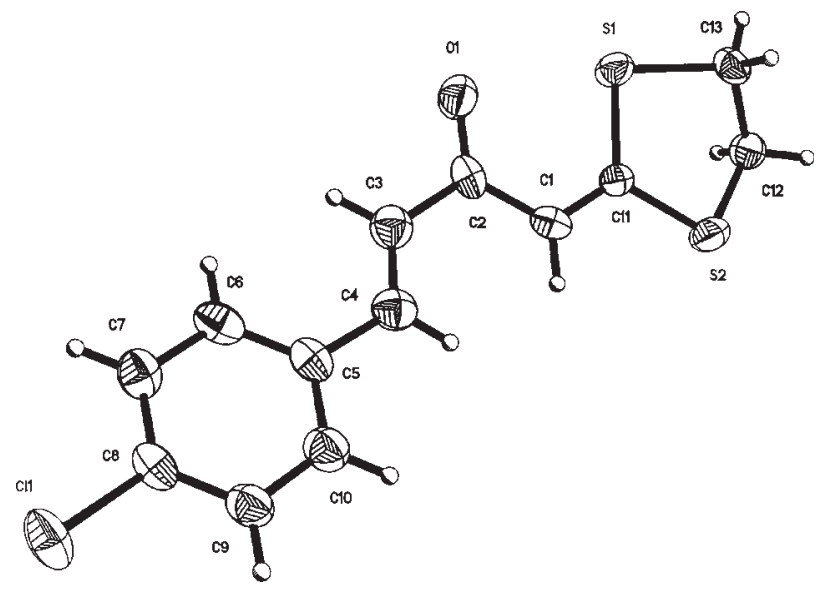

Figure 1. ORTEP drawing of ligand 3. Thermal ellipsoids at $30 \%$ probability level.

Table 1. Selected bond lengths $(\AA)$ and angles $\left({ }^{\circ}\right)$ for compound 3

\begin{tabular}{llll}
\hline S1-C11 & $1.735(3)$ & S2-C11 & $1.739(3)$ \\
O1-C2 & $1.215(3)$ & C1-C11 & $1.347(4)$ \\
C3-C4 & $1.278(4)$ & C4-C5 & $1.506(4)$ \\
S1-C13 & $1.882(12)$ & S2-C12 & $1.814(10)$ \\
S1-C13B & $1.789(11)$ & S2-C12B & $1.785(9)$ \\
C1-C2 & $1.455(4)$ & C2-C3 & $1.491(4)$ \\
C11-S1-C13 & $94.4(4)$ & C11-S2-C12 & $97.1(3)$ \\
C11-S1-C13B & $95.9(4)$ & C11-S2-C12B & $97.7(3)$ \\
C1-C11-S1 & $124.3(2)$ & C1-C11-S2 & $121.5(2)$ \\
C12-C13-S1 & $105.7(10)$ & O1-C2-C3 & $117.8(3)$ \\
C11-C1-C2 & $122.1(3)$ & O1-C2-C1 & $121.4(3)$ \\
C4-C3-C2 & $122.1(3)$ & C3-C4-C5 & $124.4(3)$ \\
S1-C11-S2 & $114.2(15)$ & C13-C12-S2 & $104.9(10)$ \\
C1-C2-C3 & $120.9(3)$ & C13B-C12B-S2 & $107.7(9)$ \\
\hline
\end{tabular}

anhydrous THF led to the formation of four complexes (Scheme 3), being 8 the major product, evidencing the well-known reductive character of $\mathrm{Fe}_{2}(\mathrm{CO})_{9} \cdot{ }^{8}$ The new $\eta^{2}$-[Fe(CO) $\left.)_{4}\right](2 \mathrm{a})$ and $\kappa^{\mathrm{N}}-\left[\mathrm{Fe}(\mathrm{CO})_{4}\right](9)$ complexes were obtained in a very low yield and the complex 7 was obtained as a result of both $\mathrm{C}-\mathrm{S}$ bond activation in the ligand in agreement with our previous studies. ${ }^{6}$ This complex has been obtained by other methods. ${ }^{9}$

When the coordination reactions were carried out with ligands 3 and 4 (Scheme 4), the formation of four complexes was observed. The expected $\eta^{2}-\left[\mathrm{Fe}(\mathrm{CO})_{4}\right]$ (3a 
<smiles>O=C(O)CC(=O)C=C1SCCS1</smiles>

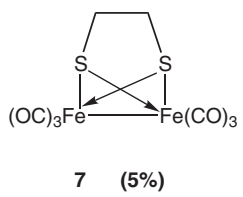<smiles>O=C(C=C1SCCS1)/C=C/c1ccc([N+](=O)[O-])cc1</smiles><smiles>Nc1ccc(/C=C/C(=O)C=C2SCCS2)cc1</smiles><smiles>O=C(C=C1SCCS1)/C=C/c1ccc(NC(=O)[O-])cc1</smiles>

Scheme 3.

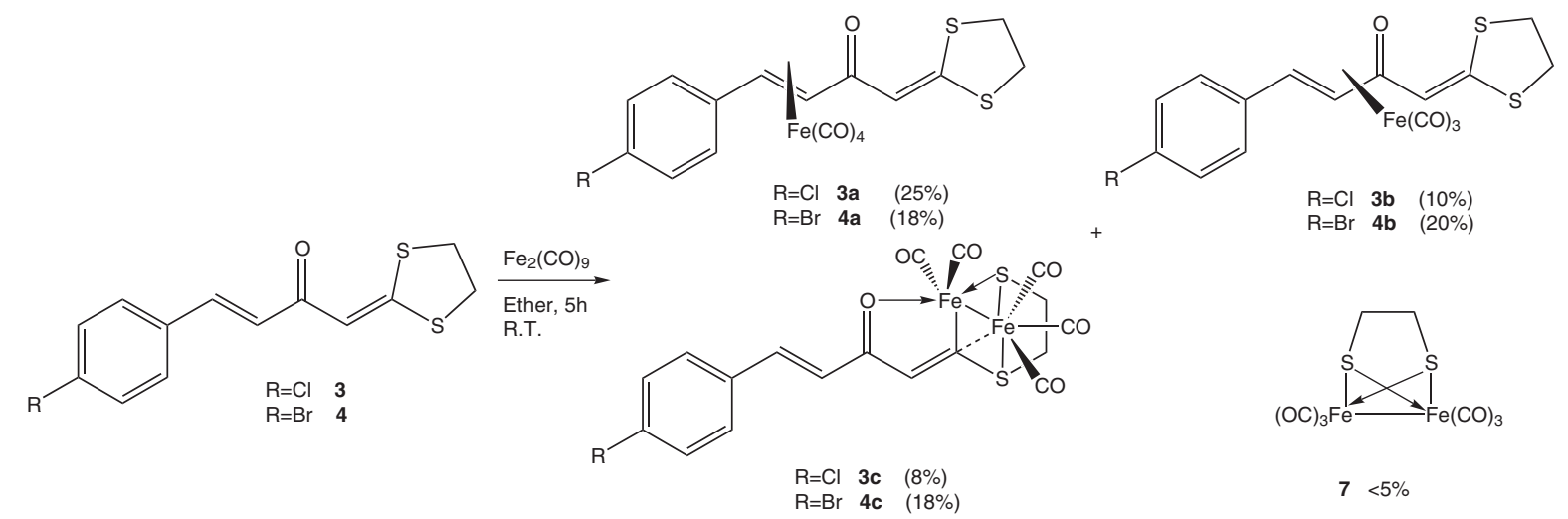

Scheme 4.

and $\mathbf{4 a}$ ) and $\eta^{4}-\left[\mathrm{Fe}(\mathrm{CO})_{3}\right]$ (3b and $\left.\mathbf{4 b}\right)$ complexes were obtained as orange crystalline solids. These complexes displayed in IR spectra 3 bands around $2000 \mathrm{~cm}^{-1}$ that correspond to $v(\mathrm{M}-\mathrm{CO})$ region. In MS the corresponding peaks assigned to the fragments $\left[\mathrm{M}^{+}-28\right]$ were observed. The ${ }^{1} \mathrm{H}$ NMR spectra for these complexes exhibit the peaks corresponding to the $\mathrm{AB}$ system shifted upfield, indicating the coordination of the iron complex to the $\mathrm{C}=\mathrm{C} \pi$-system. The chemical shift of the other signals was very similar to that of the free-ligands. The ${ }^{13} \mathrm{C}$ NMR spectra of these complexes show that the corresponding signals to the $\mathrm{C}=\mathrm{C}$ double bond are shifted to higher frequencies $(\Delta \delta$ around 67). Signals around 200 and $208 \mathrm{ppm}$ were assigned for $\mathrm{M}-\mathrm{CO}$ group. The most salient difference between $\eta^{2}$-[Fe( $\left.\left.\mathrm{CO}\right)_{4}\right]$ and $\eta^{4}-\left[\mathrm{Fe}(\mathrm{CO})_{4}\right]$ complexes is that for $\eta^{4}-\left[\mathrm{Fe}(\mathrm{CO})_{3}\right]$ complexes $(\mathbf{3 b}$ and $\mathbf{4 b})$, the ketone group signal was shifted from $184 \mathrm{ppm}$ at $155 \mathrm{ppm}$ and this fact indicates the coordination of $\mathrm{CO}$ group to iron atom.

The X-ray diffraction analysis for $\mathbf{4 b}$ (Figure 2), showed that the $\left[\mathrm{Fe}(\mathrm{CO})_{3}\right]$ fragment was bonded in $\eta^{4}$-coordination mode to the enone moiety O4-C5-C6-C7, thus the coordination polyhedron can be best described as a distorted trigonal bipyramid, with two carbonyl groups and the $\mathrm{C} 3-\mathrm{C} 4$ double bond in the equatorial positions, with the $\mathrm{O} 4$ oxygen atom and the remaining $\mathrm{CO}$ group in apical positions with a bond angle of $164.5(2)^{\circ}$ (Table 2). This arrangement is in agreement with other $\eta^{4}-\left[\mathrm{Fe}(\mathrm{CO})_{3}\right]$ complexes described in literature. ${ }^{10}$ One O4---S1 interaction $(2.757 \AA$ ) was also present and was in the same order than with the obtained crystallographic data from 3. The 1,3-dithiolane ring adopts an envelope conformation with $\mathrm{C} 16(\mathrm{C} 16 \mathrm{~B})$ as flap, the crystalline structure showed disorder in this moiety leading two conformers in 77/23 ratio, only the major contributor are shown in Figure 2.

Additionally, the dinuclear complexes $\mathbf{3 c}$ and $\mathbf{4 c}$ were obtained in moderate yields. They have the same structural arrangement as the complex 1c, previously reported. ${ }^{6,11}$ The IR spectra of these compounds show the typical bands of $v(\mathrm{M}-\mathrm{CO})$ in 2058 and $2017 \mathrm{~cm}^{-1}$. The ${ }^{1} \mathrm{H}$ NMR spectra of $\mathbf{3 c}$ and $\mathbf{4} \mathbf{c}$ exhibit changes in the assigned signals to the methylene groups, which shifted upfield with respect to ligands. In ${ }^{13} \mathrm{C}$ NMR spectra, as key signals, we observed that the alpha $\mathrm{CH}$ and the carbon attached to sulfur and iron atoms were shifted to higher frequencies (12 and 25 ppm respectively). In both reactions, complex 7 was obtained in a yield of $5 \%$. 


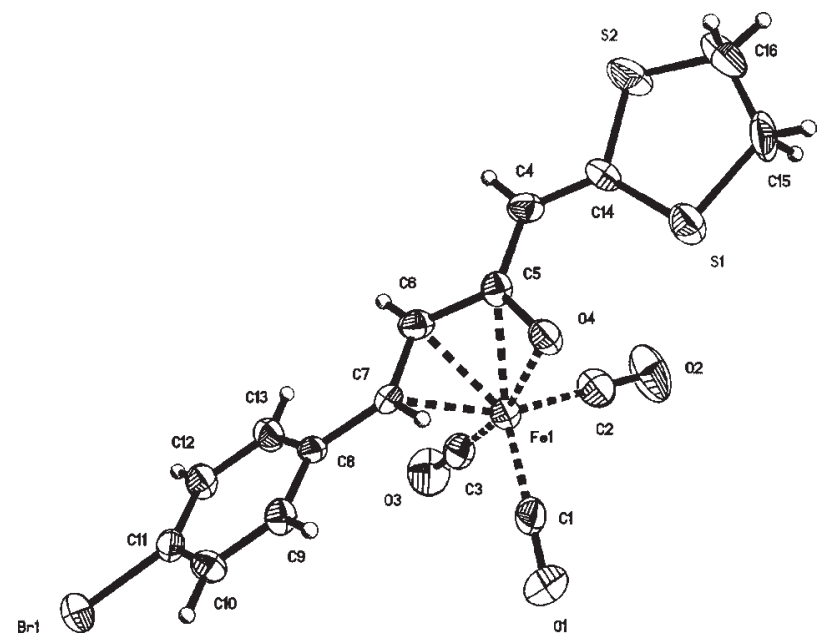

Figure 2. ORTEP drawing of ligand $\mathbf{4 b}$. Thermal ellipsoids at $30 \%$ probability level.

The reaction with ligands $\mathbf{5}$ and $\mathbf{6}$ lead to the formation of the $\eta^{4}$-[Fe( $\left.\left.\mathrm{CO}\right)_{3}\right]$ complexes $(\mathbf{5 b}$ and $\mathbf{6 b})$ in low yield (Scheme 5), being the major products the dinuclear complexes $\mathbf{5 c}$ and $\mathbf{6 c}$, these complexes displayed a similar structural arrangement to those of $\mathbf{1 c}, \mathbf{3 c}$ and $\mathbf{4 c}$ vide supra. In both experiments, complex 7 was also obtained in low yield and for the ligand $\mathbf{6}$, the $\kappa^{\mathrm{N}}$-[Fe(CO) $\left.{ }_{4}\right]$ complex (10) was additionally obtained in traces. For $\mathbf{6 b}$, an additionally $\sigma$-N coordination of $\left[\mathrm{Fe}(\mathrm{CO})_{4}\right]$ fragment towards amine group was evidenced by ${ }^{1} \mathrm{H}$ and ${ }^{13} \mathrm{C} \mathrm{NMR}$, as well as by a peak in $599 \mathrm{~m} / \mathrm{z}$ in MS.

We have carried out some experiments exploring different condition reaction (Table 4), which evidenced that the coordination reactions were sensitive to the temperature and the stability of the complexes. Thus, the
$\eta^{2}$-[Fe( $\left.\left.\mathrm{CO}\right)_{4}\right]$ complexes are intermediates in the formation of most thermodynamic stable $\eta^{4}$-[Fe( $\left.\left.\mathrm{CO}\right)_{3}\right]$ complexes, whose formation depends strongly on the electronic characteristics of the $\mathrm{C}-\mathrm{C}$ double bond. ${ }^{12}$ The dinuclear complexes $\mathbf{3 c - 6 c}$ are the intermediates in the formation of complex 7, resulting from the total C-S bond activation of the corresponding ligands, as we have previously proposed. ${ }^{6}$

Table 2. Selected bond lengths $(\AA)$ and angles $\left(^{\circ}\right)$ for compound $\mathbf{4 b}$

\begin{tabular}{|c|c|c|c|}
\hline $\mathrm{Fe} 1-\mathrm{C} 3$ & $1.745(6)$ & $\mathrm{Fe} 1-\mathrm{C} 1$ & $1.795(6)$ \\
\hline $\mathrm{Fe} 1-\mathrm{C} 2$ & $1.830(6)$ & $\mathrm{Fe} 1-\mathrm{O} 4$ & $2.014(3)$ \\
\hline $\mathrm{Fe} 1-\mathrm{C} 6$ & $2.037(4)$ & Fe1-C5 & $2.112(5)$ \\
\hline $\mathrm{Fe} 1-\mathrm{C} 7$ & $2.134(4)$ & $\mathrm{S} 1-\mathrm{C} 14$ & $1.726(5)$ \\
\hline $\mathrm{S} 1-\mathrm{C} 15$ & $1.798(12)$ & $\mathrm{S} 2-\mathrm{C} 16$ & $1.740(8)$ \\
\hline $\mathrm{S} 2-\mathrm{C} 14$ & $1.748(5)$ & $\mathrm{O} 1-\mathrm{C} 1$ & $1.153(6)$ \\
\hline $\mathrm{O} 2-\mathrm{C} 2$ & $1.124(5)$ & $\mathrm{O} 3-\mathrm{C} 3$ & $1.155(6)$ \\
\hline $\mathrm{O} 4-\mathrm{C} 5$ & $1.299(5)$ & $\mathrm{C} 4-\mathrm{C} 14$ & $1.347(6)$ \\
\hline $\mathrm{C} 4-\mathrm{C} 5$ & $1.449(6)$ & C5-C6 & $1.416(6)$ \\
\hline C6-C7 & $1.416(5)$ & $\mathrm{C} 15-\mathrm{C} 16$ & $1.397(14)$ \\
\hline S1-C15B & $1.83(4)$ & S2-C16B & $1.92(3)$ \\
\hline C15B-C16B & $1.57(5)$ & $\mathrm{C} 1-\mathrm{Fe} 1-\mathrm{C} 2$ & $104.6(2)$ \\
\hline $\mathrm{C} 3-\mathrm{Fe} 1-\mathrm{C} 1$ & $96.8(3)$ & $\mathrm{C} 3-\mathrm{Fe} 1-\mathrm{C} 2$ & $90.5(2)$ \\
\hline $\mathrm{C} 3-\mathrm{Fe} 1-\mathrm{O} 4$ & $164.5(2)$ & C3-Fe1-C6 & $97.8(2)$ \\
\hline C1-Fe1-O4 & $97.7(2)$ & $\mathrm{C} 2-\mathrm{Fe} 1-\mathrm{O} 4$ & $91.15(19)$ \\
\hline C1-Fe1-C6 & $131.3(2)$ & $\mathrm{C} 3-\mathrm{Fe} 1-\mathrm{C} 5$ & $127.9(2)$ \\
\hline $\mathrm{C} 1-\mathrm{Fe} 1-\mathrm{C} 5$ & $132.3(2)$ & C6-Fe1-C5 & $39.9(16)$ \\
\hline $\mathrm{C} 3-\mathrm{Fe} 1-\mathrm{C} 7$ & $95.9(2)$ & $\mathrm{O} 4-\mathrm{Fe} 1-\mathrm{C} 7$ & $77.96(15)$ \\
\hline C6-Fe1-C7 & $39.6(14)$ & C16-S2-C14 & $96.5(4)$ \\
\hline $\mathrm{C} 2-\mathrm{Fe} 1-\mathrm{C} 6$ & $121.44(2)$ & $\mathrm{O} 4-\mathrm{Fe} 1-\mathrm{C} 6$ & $63.36(15)$ \\
\hline $\mathrm{C} 2-\mathrm{Fe} 1-\mathrm{C} 5$ & $92.0(2)$ & O4-Fe1-C5 & $36.62(13)$ \\
\hline $\mathrm{C} 1-\mathrm{Fe} 1-\mathrm{C} 7$ & $92.8(2)$ & $\mathrm{C} 2-\mathrm{Fe} 1-\mathrm{C} 7$ & $160.7(2)$ \\
\hline $\mathrm{C} 5-\mathrm{Fe} 1-\mathrm{C} 7$ & $69.67(17)$ & C14-S1-C15 & $92.8(4)$ \\
\hline C14-C4-C5 & $123.3(5)$ & C14-S1-C15B & $108.0(14)$ \\
\hline $\mathrm{O} 1-\mathrm{C} 1-\mathrm{Fe} 1$ & $179.1(5)$ & $\mathrm{O} 2-\mathrm{C} 2-\mathrm{Fe} 1$ & $179.3(7)$ \\
\hline $\mathrm{C} 5-\mathrm{O} 4-\mathrm{Fe} 1$ & $75.8(3)$ & $\mathrm{O} 3-\mathrm{C} 3-\mathrm{Fe} 1$ & $177.6(6)$ \\
\hline O4-C5-C6 & $113.8(4)$ & $\mathrm{O} 4-\mathrm{C} 5-\mathrm{C} 4$ & $121.3(4)$ \\
\hline
\end{tabular}

Table 3. Summary of Crystal data, data collection, and refinement details

\begin{tabular}{|c|c|c|}
\hline Compound & 3 & $4 b$ \\
\hline Formula & $\mathrm{C}_{13} \mathrm{H}_{11} \mathrm{ClOS}_{2}$ & $\mathrm{C}_{16} \mathrm{H}_{11} \mathrm{BrFeO}_{4} \mathrm{~S}_{2}$ \\
\hline Molecular weight (g/mol) & 282.79 & 467.13 \\
\hline Description & Orange prism & Red prism \\
\hline crystal size (mm) & $0.308 \times 0.30 \times 0.108$ & $0.224 \times 0.09 \times 0.06$ \\
\hline crystalline system & Monoclinic & Monoclinic \\
\hline Spatial group & $\mathrm{P} 2_{1} / \mathrm{n}$ & $\mathrm{P} 2{ }_{1} / \mathrm{c}$ \\
\hline \multirow[t]{3}{*}{ Cell parameters $\left(\AA,^{\circ}\right)$} & $\mathrm{a}=6.1474(4) \quad \alpha=90$ & $\mathrm{a}=7.661(1)$ \\
\hline & $\mathrm{b}=18.839(1) \quad \beta=95.302(2)$ & $\mathrm{b}=17.609(1)$ \\
\hline & $\mathrm{c}=11.090(1)$ & $\mathrm{c}=13.252(1)$ \\
\hline Volume $\left(\AA^{3}\right)$ & $1278.9(2)$ & $1787.2(3)$ \\
\hline $\mathrm{Z}$ & 4 & 4 \\
\hline $\mathrm{D}_{\text {calc }} \cdot\left(\mathrm{Mg} / \mathrm{m}^{3}\right)$ & 1.469 & 1.736 \\
\hline No. colect. reflex. & 12640 & 17743 \\
\hline No. Ind. Reflex. & 2923 & 4107 \\
\hline Date /parameters & $2923 / 173$ & $4107 / 226$ \\
\hline$R_{\text {int }}$ & 0.0452 & 0.1069 \\
\hline R1, wR2 [I t 2 $2 \sigma(\mathrm{I})]$ & $0.0564,0.1196$ & $0.0485,0.0556$ \\
\hline R1, wR2 [all the date] & $0.0859,0.1315$ & $0.1479,0.0718$ \\
\hline GOOF in $F^{2}$ & 0.992 & 0.957 \\
\hline
\end{tabular}


Table 4. Reaction of ligands 3-6 with $\mathrm{Fe}_{2}(\mathrm{CO})_{9}$

\begin{tabular}{|c|c|c|c|c|c|c|}
\hline Condition reaction & Ligand & $\eta^{2}-\left[\mathrm{Fe}(\mathrm{CO})_{4}\right]$ & $\eta^{4}-\left[\mathrm{Fe}(\mathrm{CO})_{3}\right]$ & $\mathrm{LFe}_{2}(\mathrm{CO})_{5}$ & $\left(\mathrm{CH}_{2} \mathrm{~S}\right)_{2} \mathrm{Fe}_{2}(\mathrm{CO})_{6}(7)$ & $\mathrm{LFe}(\mathrm{CO})_{4}$ \\
\hline \multirow[t]{2}{*}{ Ether, RT, 5h } & 3 & 3a: $25 \%$ & 3b: $10 \%$ & 3c: $8 \%$ & \multirow[t]{2}{*}{$<5 \%$} & \\
\hline & 4 & 4a: $18 \%$ & 4b: $20 \%$ & 4c: $18 \%$ & & \\
\hline \multirow[t]{2}{*}{ THF, RT, 24h } & 3 & 3a: - & 3b: $25 \%$ & 3c: $7 \%$ & \multirow[t]{2}{*}{$20 \%$} & \\
\hline & 4 & 4a: - & $4 b: 32 \%$ & 4c: $10 \%$ & & \\
\hline \multirow[t]{2}{*}{ Ether, RT, 5h } & 5 & 5a: - & 5b: $20 \%$ & 5c: $28 \%$ & \multirow[t]{2}{*}{$<5 \%$} & \multirow[t]{2}{*}{ 10: $2 \%$} \\
\hline & 6 & 6a: - & 6b: $7 \%$ & 6c: $25 \%$ & & \\
\hline \multirow[t]{2}{*}{ THF, RT, 24h } & 5 & $5 a:-$ & 5b: $18 \%$ & 5c: $12 \%$ & \multirow[t]{2}{*}{$18 \%$} & \multirow[t]{2}{*}{$10:-$} \\
\hline & 6 & 6a: - & 6b: $5 \%$ & 6c: $10 \%$ & & \\
\hline Refluxing THF, 24h & 6 & 15a: - & 6b: detected by TLC & 6c: $<5 \%$ & $27 \%$ & 10: - \\
\hline Ether, $5^{\circ} \mathrm{C}, 24 \mathrm{~h}$ & 6 & 15a: - & 6b: $13 \%$ & 6c: $28 \%$ & - & 10: $5 \%$ \\
\hline
\end{tabular}

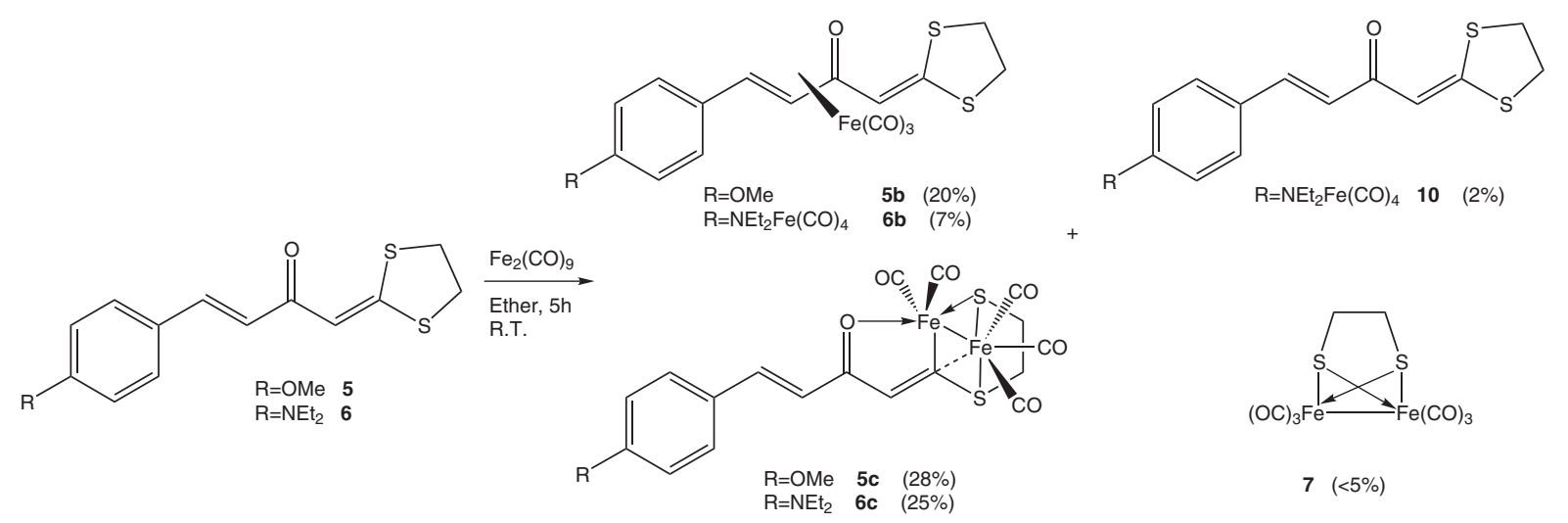

Scheme 5.

Screening the reactivity of $\mathrm{Fe}_{2}(\mathrm{CO})_{9}$ toward this kind of systems, the chemical shift of C-3 on ${ }^{13} \mathrm{C}$ NMR have been analyzed using correlation with Hammett type substituent constant $\left(\sigma_{\mathrm{p}}\right)^{13}$ (Table 5) giving a lineal correlation as shown in Figure 3.

The positive value of $(\rho)$ indicates that the electronic effect of the group on the aromatic ring increased the electronic density over $\mathrm{C} 3$ as a long distance $\beta$ effect (Scheme 6). ${ }^{16}$

As a result of this study, and the several possibility of coordination ability of $\mathrm{Fe}(0)$ towards cross-conjugated compounds, we have observed that $\left[\mathrm{Fe}(\mathrm{CO})_{4}\right]$ fragment has a preference to react with electronic deficient double bound in agreement with G. Reichenbach, ${ }^{17}$ who proposed that the $\left[\mathrm{Fe}(\mathrm{CO})_{4}\right]$ fragment is an electron-relaxing. Thus, when there are electron-withdrawing groups on the aromatic ring, the reaction produce the $\eta^{2}$-[Fe( $\left.\left.\mathrm{CO}\right)_{4}\right]$ and $\eta^{4}$-[ $\left.\mathrm{Fe}(\mathrm{CO})_{4}\right]$ complexes as the main compounds, in agreement with the Hammett plot.

Nevertheless, with electron-donating groups (Scheme 5 ), it might be expected that the transmission of electronic effects should increased by mesomeric effect over $\mathrm{C} 3=\mathrm{C} 4$ and then, the reaction proceeds differently leading as major products the dinuclear complexes resulting from the carbon-sulfur bond activation.

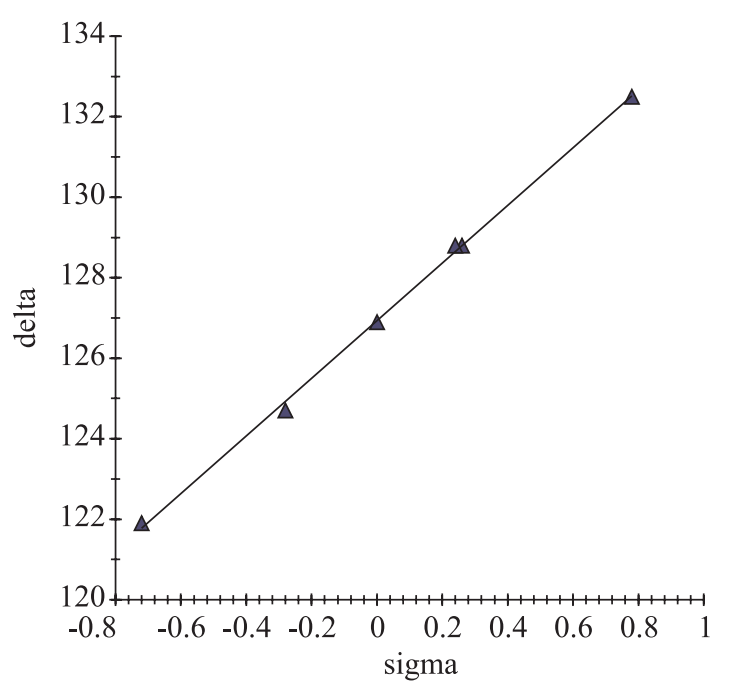

Figure 3. Hammett correlation, $\delta=7.1594 \sigma+126.93, \mathrm{r}=0.9993$.

Table 5. Chemical shift on ${ }^{13} \mathrm{C}$ NMR from $\mathrm{C} 3$ and $\sigma_{p}$

\begin{tabular}{llll}
\hline Ligand & $\mathrm{R}$ & $\delta \mathrm{C} 3$ & $\sigma_{p}$ \\
\hline 2 & $p-\mathrm{NO}_{2}$ & 132.5 & $0.78^{14}$ \\
3 & $p-\mathrm{Br}$ & 128.8 & $0.26^{15}$ \\
4 & $p-\mathrm{Cl}$ & 128.8 & $0.24^{15}$ \\
1 & $p-\mathrm{H}$ & 126.9 & 0 \\
5 & $p-\mathrm{OMe}$ & 124.74 & $-0.28^{15}$ \\
6 & $p-\mathrm{N}(\mathrm{Et})_{2}$ & 121.9 & $-0.72^{14}$ \\
\hline
\end{tabular}




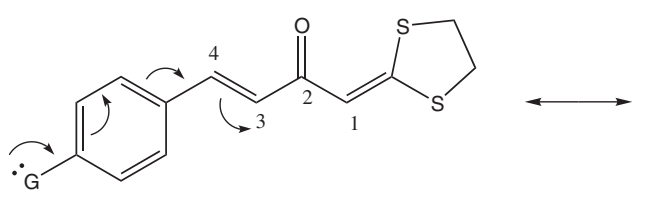<smiles>O=C1C=CC(=CC(=O)C=C2SCCS2)C=C1</smiles>

Scheme 6.

\section{Conclusion}

In this work, we have evidenced that the reactivity of $\mathrm{Fe}_{2}(\mathrm{CO})_{9}$ with cross-conjugated carbonyl ligands depends on the electronic density of the heterodienic moiety, thus, electron-withdrawing groups promote the coordination reaction towards the formation of $\eta^{2}-\left[\mathrm{Fe}(\mathrm{CO})_{4}\right]$ and $\eta^{4}$ - $\left[\mathrm{Fe}(\mathrm{CO})_{3}\right]$ complexes, while electron-donor groups aim the reactivity of $\mathrm{Fe}_{2}(\mathrm{CO})_{9}$ towards the heterodienic moiety $\beta$-positioned by sulfur atoms leading the formation of dinuclear iron complexes, coming from the partial and total carbon-sulfur bonds activation.

\section{Experimental}

${ }^{1} \mathrm{H} \mathrm{NMR}$ and ${ }^{13} \mathrm{C}$ NMR spectra were recorded on a JEOL 300 spectrometer, using $\mathrm{CDCl}_{3},\left(\mathrm{CD}_{3}\right)_{2} \mathrm{SO}$ and $\mathrm{C}_{6} \mathrm{D}_{6}$ as solvents and TMS as internal reference. IR spectra were performed on a Perkin-Elmer 283 B or 1420 spectrometer. The FAB spectra were obtained on a JEOL JMS SX 102A mass spectrometer operated at an accelerating voltage of $10 \mathrm{Kv}$. Samples were desorbed from a nitrobenzyl alcohol matrix using a $6 \mathrm{kev}$ Xenon atoms. The electronic impact (EI) ionization mass spectra were acquired on a JEOL JMSAX505 HA Mass spectrometer operated in the positive ion mode. The acquisition conditions were ion source temperature $230{ }^{\circ} \mathrm{C}$, ionization energy $70 \mathrm{eV}$, emission current $0.14 \mathrm{~mA}$ and ionization current $100 \mathrm{~mA}$. Mass measurements in FAB are performed at 10000 resolution using electrical field scans and the polyethylene glycol ions as reference material. Melting points were measured using a Mel-Temp II apparatus and are uncorrected. All reagents were obtained from commercial suppliers and used as received. Reactions were performed under nitrogen atmosphere in carefully dried glassware. THF and ether were distilled from sodium-benzophenone under argon and/or nitrogen atmosphere. Column chromatography was performed with Merck silica gel (70-230 mesh) or alumina using ethyl acetate: hexane in different ratios as eluent.

\section{Synthesis of ligands}

To a solution of $5 \mathrm{mmol}$ of 1-(1,3-dithiolane-2-yliden) acetone $^{6}$ in $20 \mathrm{~mL}$ of ethanol was added $10 \mathrm{~mL}$ of an alcoholic solution of $\mathrm{NaOH}(5.5 \mathrm{mmol})$ at $0{ }^{\circ} \mathrm{C}$. Then, a solution of corresponding aldehyde $(5 \mathrm{mmol})$ in $5 \mathrm{~mL}$ of ethanol was added and the reaction was keeping on magnetic stirring during $18 \mathrm{~h}$ at room temperature. The solvent was eliminated for distillation at reduced pressure, then Ethyl acetate $(50 \mathrm{~mL})$ and water $(2 \times 50 \mathrm{~mL})$ were also added, extracting the organic phase, drying with $\mathrm{Na}_{2} \mathrm{SO}_{4}$ and removing the volatile in vacuo. The reaction mixture was purified by column chromatography on alumina, using hexane/AcOEt in different ratios as eluent.

\section{Synthesis of complexes}

A solution of the ligand 2-6 (1 mmol) in anhydrous THF or Ether $(40 \mathrm{~mL})$ was treated with $\mathrm{Fe}_{2}(\mathrm{CO})_{9}(3 \mathrm{mmol})$ with magnetic stirring at room temperature for $5 \mathrm{~h}$ under inert atmosphere. After the reaction was complete, the crude was filtered off through an alumina column and the solvent was evaporated under vacuum. The reaction mixture was chromatographed on silica gel and elution with hexane/ ethyl acetate in different ratio gave the corresponding $\mathrm{Fe}$ (0) complexes. The yields were based on the pure products isolated.

\section{Ligands}

4-[p-Nitrophenyl]-1-(1,3-dithiolan-2-ylidene)-3Ebuten-2-one, (2). Purification: CC, Hexane/AcOEt, 5:5. Yellow solid, (40\%), m.p. $128^{\circ} \mathrm{C}$; ${ }^{1} \mathrm{H}$ NMR $(300 \mathrm{MHz}$, $\left.\left(\mathrm{CD}_{3}\right)_{2} \mathrm{SO}\right): \delta 3.48\left(\mathrm{~m}, 4 \mathrm{H}, \mathrm{CH}_{2} \mathrm{~S}\right), 6.46$ (s, 1H, CHCSS), 7.13 and 7.63 (AB system, $2 \mathrm{H}, J 16.10 \mathrm{~Hz}, \mathrm{CH}=\mathrm{CH}$ ), 7.93 and 8.23 (AA'BB' system, $4 \mathrm{H}, J 8.81 \mathrm{~Hz}, \mathrm{CH}_{\text {arom }}$ ). ${ }^{13} \mathrm{C} \mathrm{NMR}$ (75 MHz, $\left.\left(\mathrm{CD}_{3}\right)_{2} \mathrm{SO}\right): \delta 35.5\left(\mathrm{CH}_{2} \mathrm{~S}\right), 40.4\left(\mathrm{CH}_{2} \mathrm{~S}\right), 104.9$ $\left(\underline{\mathrm{C}}_{i} \mathrm{CH}\right), 112.0$ (ㄷCSS), $124.9\left(\underline{\mathrm{CH}}_{o} \mathrm{C}_{i} \mathrm{NO}_{2}\right), 129.4$ ( $\left.\underline{\mathrm{CH}}_{o} \mathrm{C}_{i} \mathrm{CH}\right), 132.5$ (때CO), 139.6 ( $\left.\mathrm{Ph} \underline{\mathrm{CH}}\right), 146.8\left(\underline{\mathrm{C}}_{i} \mathrm{NO}_{2}\right)$, $169.5(\mathrm{CSS}), 184.3(\mathrm{CO})$. IR $\left(\mathrm{CHCl}_{3}\right) v_{\text {máx }} / \mathrm{cm}^{-1}: 1594(\mathrm{C}=\mathrm{O})$, $1499(\mathrm{C}=\mathrm{C}), 1440\left(\mathrm{NO}_{2}\right)$. M.S. (E.I. $\left.70 \mathrm{eV}\right) \mathrm{m} / \mathrm{z}(\%)$ : $\left[\mathrm{M}^{+}\right](293), 265(90), 145(45)$.

4-(p-Clorophenyl)-1-(1,3-dithiolan-2-ylidene)-3Ebuten-2-one, (3). Purification: CC, Hexane/AcOEt, 6:4. Pale yellow solid, (78\%), m.p. $119^{\circ} \mathrm{C} .{ }^{1} \mathrm{H}$ NMR $(300 \mathrm{MHz}$, $\mathrm{CDCl}_{3}$ ): $\delta 3.48\left(\mathrm{~m}, 2 \mathrm{H}, \mathrm{CH}_{2} \mathrm{~S}\right), 3.94\left(\mathrm{~m}, 2 \mathrm{H}, \mathrm{CH}_{2} \mathrm{~S}\right), 6.83(\mathrm{~s}$, 1H, CHCSS), 6.75 and 7.57 (AB system, 2H, $J 15.9 \mathrm{~Hz}$, $\mathrm{CH}=\mathrm{CH}$ ), 7.34 and 7.48 (AA'BB' system, 4H, J 8.5 Hz, 
$\left.\mathrm{CH}_{\text {arom }}\right) \cdot{ }^{13} \mathrm{C} \mathrm{NMR}\left(75 \mathrm{MHz}, \mathrm{CDCl}_{3}\right): \delta 35.5\left(\mathrm{CH}_{2} \mathrm{~S}\right), 39.1$

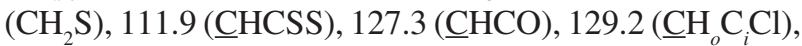
129.4 ( $\left.\underline{\mathrm{CH}}_{o} \mathrm{CH}\right), 135.9\left(\underline{\mathrm{C}}_{i} \mathrm{CH}\right), 137.8\left(\underline{\mathrm{C}}_{i} \mathrm{Cl}\right), 140.1$ ( $\left.\mathrm{Ph} \underline{\mathrm{CH}}\right)$, 167.5 (CSS), $184.3(\mathrm{CO})$. IR $\left(\mathrm{CHCl}_{3}\right) v_{\text {máx }} / \mathrm{cm}^{-1}: 1633(\mathrm{C}=\mathrm{O})$, $1493(\mathrm{C}=\mathrm{C})$. M.S. $\left(\mathrm{FAB}^{+} 70 \mathrm{eV}\right) \mathrm{m} / \mathrm{z}(\%):\left[\mathrm{M}^{+}+1\right] 283(100)$, 154(5), 136(35).

4-(p-Bromophenyl)-1-(1,3-dithiolan-2-ylidene)-3Ebuten-2-one, (4). Purification: CC, Hexane/AcOEt, 6:4. Pale yellow solid, (80\%) m.p. 145-7 ${ }^{\circ} \mathrm{C} .{ }^{1} \mathrm{H}$ NMR (300 $\left.\mathrm{MHz},\left(\mathrm{CD}_{3}\right)_{2} \mathrm{SO}\right): \delta 3.47\left(\mathrm{~m}, 4 \mathrm{H}, \mathrm{CH}_{2} \mathrm{~S}\right), 6.42(\mathrm{~s}, 1 \mathrm{H}$, CHCSS), 6.97 and 7.52 (AB system, 2H, J 16.1 Hz, $\mathrm{CH}=\mathrm{CH}$ ), 7.18 y 7.41 (AA'BB' system, 4H, J $8.26 \mathrm{~Hz}$, $\left.\mathrm{CH}_{\text {arom }}\right) \cdot{ }^{13} \mathrm{C} \mathrm{NMR}\left(75 \mathrm{MHz},\left(\mathrm{CD}_{3}\right)_{2} \mathrm{SO}\right): \delta 35.8\left(\mathrm{CH}_{2} \mathrm{~S}\right), 39.3$

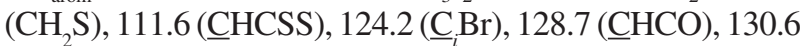
$\left(\underline{\mathrm{CH}}_{o} \mathrm{C}_{i} \mathrm{CH}\right), 132.5\left(\underline{\mathrm{CH}}_{o} \mathrm{C} \mathrm{Br}\right), 134.8\left(\underline{\mathrm{C}}_{i} \mathrm{CH}\right), 139.7(\mathrm{PhCH})$, 168.1 (CSS), 184.5 (CO). IR $\left(\mathrm{CHCl}_{3}\right) v_{\text {máx }} / \mathrm{cm}^{-1}: 1633(\mathrm{C}=\mathrm{O})$, 1489(C=C). M.S. (E.I. $70 \mathrm{eV}) \mathrm{m} / z(\%): 328\left[\mathrm{M}^{+}\right](62)$, 300(52), 216(46), 145(100).

4-(p-Methoxiphenyl)-1-(1,3-dithiolan-2-ylidene)-3Ebuten-2-one, (5). Purification: CC, Hexane/AcOEt, 85:15. Yellow crystalline solid (64\%), m.p. 101-103 ${ }^{\circ} \mathrm{C} .{ }^{1} \mathrm{H}$ NMR (300 MHz, $\left.\mathrm{CDCl}_{3}\right): \delta 3.34\left(\mathrm{~m}, 2 \mathrm{H}, \mathrm{CH}_{2} \mathrm{~S}\right), 3.44(\mathrm{~m}, 2 \mathrm{H}$, $\mathrm{CH}_{2} \mathrm{~S}$ ), 3.82 (s, 3H, $\mathrm{CH}_{3} \mathrm{O}$ ), 6.82 (s, 1H, CHCSS), 6.65 and 7.58 (AB system, $2 \mathrm{H}, J 15.82 \mathrm{~Hz}, \mathrm{CH}=\mathrm{CH}$ ), 6.88 and 7.49 (AA'BB' system, $\left.4 \mathrm{H}, J 8.81 \mathrm{~Hz}, \mathrm{CH}_{\text {arom }}\right) .{ }^{13} \mathrm{C} \mathrm{NMR}(75 \mathrm{MHz}$, $\left.\mathrm{CDCl}_{3}\right): \delta 35.5\left(\mathrm{CH}_{2} \mathrm{~S}\right), 39.0\left(\mathrm{CH}_{2} \mathrm{~S}\right), 55.5\left(\mathrm{CH}_{3} \mathrm{O}\right), 112.1$

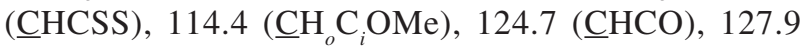
$\left(\underline{\mathrm{C}}_{i} \mathrm{CH}\right), 129.9\left(\underline{\mathrm{CH}}_{o} \mathrm{C}_{i} \mathrm{CH}\right), 141.4(\mathrm{Ph} \underline{\mathrm{CH}}), 161.3\left(\underline{\mathrm{C}}_{i} \mathrm{OCH}_{3}\right)$, 166.1 (CSS), 184.8 (CO). IR $\left(\mathrm{CHCl}_{3}\right) v_{\text {máx }} / \mathrm{cm}^{-1}: 1642(\mathrm{C}=\mathrm{O})$, 1588, 1573 and $1507(\mathrm{C}=\mathrm{C})$. M.S. (E.I. $70 \mathrm{eV}) \mathrm{m} / \mathrm{z}(\%)$ : $278\left[\mathrm{M}^{+}\right](100), 250(55), 206(20), 166(95)$.

4-[p-(N,N-diethylamino)-phenyl]-1-(1,3-dithiolan-2ylidene)-3E-buten-2-one, (6). Purification: $\mathrm{CC}$, Hexane/ AcOEt, 8:2. Red crystalline solid (68\%), m.p. 126-127 ${ }^{\circ} \mathrm{C}$. ${ }^{1} \mathrm{H} \mathrm{NMR}\left(300 \mathrm{MHz}, \mathrm{CDCl}_{3}\right): \delta 1.17\left(\mathrm{t}, 6 \mathrm{H}, \mathrm{C}_{3} \mathrm{CH}_{2} \mathrm{~N}\right), 3.38$ (q, $4 \mathrm{H}, \mathrm{CH}_{2} \mathrm{~N}$ ), 3.38 (m, 4H, $\mathrm{CH}_{2} \mathrm{~S}$ ), 6.82 (s, 1H, CHCSS), 6.57 and 7.57 (AB system, $2 \mathrm{H}, J 15.8 \mathrm{~Hz}, \mathrm{CH}=\mathrm{CH}$ ), 6.62 and 7.42 (AA'BB' system, $4 \mathrm{H}, J 8.8 \mathrm{~Hz}, \mathrm{CH}_{\text {arom }}$ ). ${ }^{13} \mathrm{C} \mathrm{NMR}$ $\left(75 \mathrm{MHz}, \mathrm{CDCl}_{3}\right): \delta 12.7\left(\underline{\mathrm{CH}}_{3} \mathrm{CH}_{2} \mathrm{~N}\right), 35.2\left(\mathrm{CH}_{2} \mathrm{~S}\right), 39.2$ $\left(\mathrm{CH}_{2} \mathrm{~S}\right), 44.8\left(\mathrm{CH}_{2} \mathrm{~N}\right), 111.6\left(\underline{\mathrm{CH}}_{o} \mathrm{C}_{i} \mathrm{~N}\right), 112.7(\underline{\mathrm{C}} \mathrm{HCSS})$, 121.9 ( $\underline{\mathrm{CHCO}}), 122.4\left(\underline{\mathrm{C}}_{i} \mathrm{CH}\right), 130.5\left(\underline{\mathrm{CH}}_{o} \mathrm{C} \mathrm{CH}\right), 142.6$ (PhCH), $149.3\left(\underline{\mathrm{C}}_{i} \mathrm{~N}\right), 167.9(\mathrm{CSS}), 185.1(\mathrm{CO}) . \mathrm{IR}\left(\mathrm{CHCl}_{3}\right)$ $v_{\text {máx }} / \mathrm{cm}^{-1}: 1632.4(\mathrm{C}=\mathrm{O}), 1565,1517$ y $1496(\mathrm{C}=\mathrm{C})$. M.S. (E.I. $70 \mathrm{eV}) \mathrm{m} / \mathrm{z}(\%): 319\left[\mathrm{M}^{+}\right]$(100), 304(90), 291(20).

\section{Complexes}

$\eta^{2}$-[4-(p-Nitrophenyl)-1-(1,3-dithiolan-2-ylidene)-3Ebuten-2-one $\mathrm{Fe}(\mathrm{CO})_{4},(2 a)$. Purification: $\mathrm{CC}$, Hexane/ AcOEt, 98:2. Orange cristalin solid (2\%), m.p. $130{ }^{\circ} \mathrm{C}$ (dec). IR $\left(\mathrm{CHCl}_{3}\right) v_{\text {máx }} / \mathrm{cm}^{-1}: 2928\left(\mathrm{Csp}^{2}-\mathrm{H}\right), 2068,2011$ y 1990
(CO-M), $1598(\mathrm{C}=\mathrm{O}), 1520(\mathrm{C}=\mathrm{C}), 1343\left(\mathrm{NO}_{2}\right)$. M.S.-E.I. $(70 \mathrm{eV}) \mathrm{m} / \mathrm{z}(\%): 461\left[\mathrm{M}^{+}\right](5), 433(8)\left(\mathrm{M}^{+}-\mathrm{CO}\right), 377(10)\left(\mathrm{M}^{+}-\right.$ 3CO), 293(100) [( $\left.\mathrm{M}^{+}-\mathrm{Fe}(\mathrm{CO})_{4}\right]$.

$\eta^{2}$-[4-(p-Clorophenyl)-1-(1,3-dithiolan-2-ilidene)-3Ebuten-2-one $\mathrm{Fe}(\mathrm{CO})_{4},(\mathbf{3 a})$. Purification: $\mathrm{CC}$, Hexane/ AcOEt, 98:2. Red cristalin solid (25\%), m.p.:95 ${ }^{\circ} \mathrm{C}$ (dec). $\left.{ }^{1} \mathrm{H} \mathrm{NMR} \mathrm{(300} \mathrm{MHz}, \mathrm{CDCl}_{3}\right): \delta 3.43\left(\mathrm{~m}, 4 \mathrm{H}, \mathrm{CH}_{2}\right), 5.85$ (s,

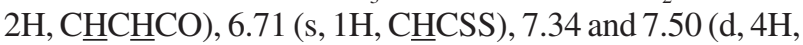
$\mathrm{C}_{\text {arom }}$ ). ${ }^{13} \mathrm{C} \mathrm{NMR}\left(75 \mathrm{MHz}, \mathrm{CDCl}_{3}\right.$ ):d 35.9 and 39.4

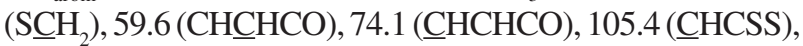
$127.9\left(\underline{\mathrm{CH}}_{o} \mathrm{C} \mathrm{Cl}\right), 129.0\left(\underline{\mathrm{CH}}_{o} \mathrm{C}_{i} \mathrm{CH}\right), 131.7\left(\underline{\mathrm{C}}_{i} \mathrm{Cl}\right) ; 138.4$ $\left(\underline{\mathrm{C}}_{\mathrm{ipso}} \mathrm{CH}\right), 182.4$ (ㅇ), 202.9 (M-CO). IR $\left(\mathrm{CHCl}_{3}\right) v_{\max } / \mathrm{cm}^{-1}$ : 2095, 2019, 1989 (M-CO), 1608 (CO). M.S.-FAB $(\mathrm{m} / \mathrm{z}): 422$ $\left(\mathrm{M}^{+}-\mathrm{CO}\right), 394\left(\mathrm{M}^{+}-2 \mathrm{CO}\right), 282\left[\mathrm{M}^{+}-\mathrm{Fe}(\mathrm{CO})_{4}\right]$. H.R.-M.S. $\left(\mathrm{FAB}^{+}\right) \mathrm{C}_{16} \mathrm{H}_{12} \mathrm{O}_{4} \mathrm{ClS}_{2} \mathrm{Fe}$, Exp.: 422.9207, Calc.: 422.9215.

$\eta^{4}$-[4-( $p$-Clorophenyl)-1-(1,3-dithiolan-2-ylidene)-3Ebuten-2-one $\mathrm{Fe}(\mathrm{CO})_{3,}(\mathbf{3 b})$. Purification: CC, Hexane/ AcOEt, 96:4. Orange cristalin solid (10\%), m.p.:128-130 ${ }^{\circ} \mathrm{C}$ (dec). ${ }^{1} \mathrm{H}$ NMR (300 MHz, $\left.\mathrm{C}_{6} \mathrm{D}_{6}\right): \delta 2.39\left(\mathrm{~m}, 4 \mathrm{H}, \mathrm{CH}_{2}\right), 3.25$

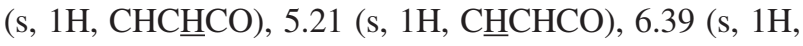
$\mathrm{C} \underline{\mathrm{H}}=\mathrm{CSS}), 6.74$ and $6.92\left(\mathrm{~d}, 4 \mathrm{H}, J 7.29 \mathrm{~Hz} \mathrm{C}_{\text {arom }}\right) .{ }^{13} \mathrm{C}$ NMR $\left(75 \mathrm{MHz}, \mathrm{C}_{6} \mathrm{D}_{6}\right): \delta 35.2$ and $38.8\left(\mathrm{SCH}_{2}\right), 59.6$

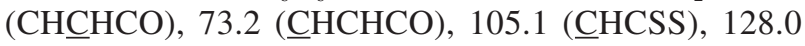
$\left(\underline{\mathrm{CH}}_{o} \mathrm{C} \mathrm{Cl}\right), 128.8\left(\underline{\mathrm{CH}}_{o} \mathrm{C}_{i} \mathrm{CH}\right), 132.1\left(\underline{\mathrm{C}}_{i} \mathrm{Cl}\right), 138.2\left(\underline{\mathrm{C}}_{i} \mathrm{CH}\right)$, 143.0 (ㄷS), 155.4 (ㅁ), 212.2 (M-CO). IR $\left(\mathrm{CHCl}_{3}\right)$ $v_{\max } / \mathrm{cm}^{-1}:$ 2064, 2006, 1986 (M-CO), 1601 (CO). M.S.-E.I. $(\mathrm{m} / \mathrm{z}): 394\left(\mathrm{M}^{+}-\mathrm{CO}\right), 338\left(\mathrm{M}^{+}-3 \mathrm{CO}\right), 282\left[\mathrm{M}^{+}-\mathrm{Fe}(\mathrm{CO})_{3}\right]$.

[4-(p-Clorophenyl)-1-(1,3-dithiolan-2-ylidene)-3Ebuten-2-one $\mathrm{Fe}_{2}(\mathrm{CO})_{5}$, (3c). Purification: $\mathrm{CC}$, Hexane/ AcOEt, 9:1. Deep red solid (8\%), m.p.:118 ${ }^{\circ} \mathrm{C}(\mathrm{dec}) .{ }^{1} \mathrm{H}$ NMR $\left(300 \mathrm{MHz}, \mathrm{CDCl}_{3}\right): \delta 2.34\left(\mathrm{~m}, 2 \mathrm{H}, \mathrm{FeSCH}_{2}\right), 3.34(\mathrm{~m}, 2 \mathrm{H}$, $\mathrm{SCH}_{2}$ ), 7.08 (s, 1H, $\mathrm{CH}=\mathrm{CSFe}$ ), 6.81 and 7.57 (AB system, $2 \mathrm{H}, J 15.98 \mathrm{~Hz}, \mathrm{C} \underline{\mathrm{HC}} \underline{\mathrm{HCO}}$ ), 7.53 and 7.71 (AA'BB' system, $\left.4 \mathrm{H}, J 8.92 \mathrm{~Hz} \mathrm{C}_{\text {arom }}\right) .{ }^{13} \mathrm{C} \mathrm{NMR}\left(75 \mathrm{MHz}, \mathrm{CDCl}_{3}\right): \delta 30.4$ $\left(\mathrm{FeSCH}_{2}\right), 32.0\left(\mathrm{~S}_{2} \mathrm{H}_{2}\right), 132.6\left(\mathrm{C}_{i}-\mathrm{Cl}\right), 114.2$ ( $\left.\underline{\mathrm{C} H C S F e}\right)$, 128.9 and $129.2\left(\underline{\mathrm{CH}}_{a r}\right), 127.8(\mathrm{CH} \underline{\mathrm{CHCO}}), 139.4$

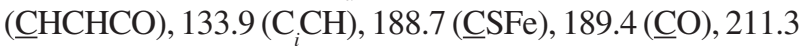
and 213.9 (M-CO) ppm. IR $\left(\mathrm{CHCl}_{3}\right) v_{\text {max }} / \mathrm{cm}^{-1}: 2065,2027$ (M-CO). M.S.-FAB ${ }^{+}(\mathrm{m} / \mathrm{z}): 478\left(\mathrm{M}^{+}-2 \mathrm{CO}\right), 339$ [( $\left.\mathrm{M}^{+}+1\right)-$ $\left.\mathrm{Fe}(\mathrm{CO})_{5}\right]$.

$\eta^{2}$-[4-(p-Bromophenyl)-1-(1,3-dithiolan-2-ylidene)-3Ebuten-2-one $\mathrm{Fe}(\mathrm{CO})_{3}$, (4a). Purification: $\mathrm{CC}$, Hexane/ AcOEt, 99:1. Red solid (18\%), m.p.:156-8 ${ }^{\circ} \mathrm{C}$ (dec); ${ }^{1} \mathrm{H}$ NMR (300 MHz, $\left.\mathrm{CDCl}_{3}\right): \delta 3.38\left(\mathrm{~m}, 4 \mathrm{H}, \mathrm{CH}_{2}\right) ; 5.85(\mathrm{AB}$ system, $1 \mathrm{H}, J 11.25 \mathrm{~Hz}, \underline{\mathrm{CH}} \underline{\mathrm{HCO}}), 6.72(\mathrm{~s}, 1 \mathrm{H}, \mathrm{C} \underline{\mathrm{H}}=\mathrm{CSS})$, 7.19 and 7.38 (AA'BB' system, $4 \mathrm{H}, J 6.33 \mathrm{~Hz} \underline{\mathrm{CH}}_{\text {arom }}$ ). ${ }^{13} \mathrm{C}$ NMR $\left(75 \mathrm{MHz}, \mathrm{CDCl}_{3}\right): \delta 31.1$ and $36.3\left(\mathrm{SCH}_{2}\right), 56.4$ (CHㅡHCO), 65.9 (대CHCO), 105.6 (ㄷCSS), 123.3 $\left(\underline{\mathrm{C}}_{i} \mathrm{Br}\right), 129.6\left(\underline{\mathrm{CH}}_{o} \mathrm{C}_{i} \mathrm{CH}\right), 132.1\left(\underline{\mathrm{CH}}_{o} \mathrm{C}_{i} \mathrm{Br}\right), 138.7\left(\underline{\mathrm{C}}_{i} \mathrm{CH}\right)$, 163.8 (ㄷSS), 190.8 (ㅁ), 200.5 and 207.5 (M-CO). IR $\left(\mathrm{CHCl}_{3}\right) v_{\max } / \mathrm{cm}^{-1}:$ 2098, 2016, 1985 (M-CO), 1589 (CO). 
M.S.-FAB ${ }^{+}(\mathrm{m} / \mathrm{z}): 495\left[\mathrm{M}^{+}\right], 467$ and $469\left(\mathrm{M}^{+}-\mathrm{CO}\right), 411$ and $412\left[\mathrm{M}^{+}-3 \mathrm{CO}\right], 327$ and $329\left[\mathrm{M}^{+}-\mathrm{Fe}(\mathrm{CO})_{4}\right]$.

$\eta^{4}$-[4-(p-Bromophenyl)-1-(1,3-dithiolan-2-ylidene)-3Ebuten-2-one] $\mathrm{Fe}(\mathrm{CO})_{3}$, (4b). Purificación: CC, Hexane/ AcOEt, 97:3. Orange solid (20\%), p.f.:83 ${ }^{\circ} \mathrm{C}(\mathrm{dec}) .{ }^{1} \mathrm{H}$ NMR $\left(300 \mathrm{MHz}, \mathrm{C}_{6} \mathrm{D}_{6}\right): \delta 2.36\left(\mathrm{~m}, 4 \mathrm{H}, \mathrm{CH}_{2}\right), 3.22$ and $5.18(\mathrm{AB}$ system, $1 \mathrm{H}, J 8.67 \mathrm{~Hz}, \mathrm{C} \underline{\mathrm{HC}} \underline{\mathrm{HCO}}), 6.38$ (s, 1H, C$=\mathrm{H}=\mathrm{CSS}$ ), 6.64 and 7.07 (AA'BB' system, $4 \mathrm{H}, J 7.29 \mathrm{~Hz} \underline{\mathrm{CH}}_{\text {arom }}$ ). ${ }^{13} \mathrm{C}$ NMR $\left(75 \mathrm{MHz}, \mathrm{C}_{6} \mathrm{D}_{6}\right): \delta 35.3$ and $38.8\left(\mathrm{SCH}_{2}\right), 59.7$

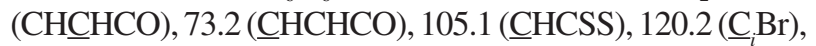
$128.1\left(\underline{\mathrm{CH}}_{o} \mathrm{C} \mathrm{CH}\right), 131.7\left(\underline{\mathrm{CH}}_{o} \mathrm{CBr}\right), 138.7\left(\underline{\mathrm{C}}_{i} \mathrm{CH}\right), 143.1$ (CSS), 155.5 (므), 200.1 (M-CO). IR (KBr) $v_{\max } / \mathrm{cm}^{-1}: 2060$, 2002, 1982 (M-CO), 1543 (CO). M.S.-FAB ${ }^{+}(\mathrm{m} / \mathrm{z}): 469$ and 467[ $\left.\mathrm{M}^{+}+1\right], 440$ and $438\left(\mathrm{M}^{+}-\mathrm{CO}\right) ; 411$ and $412\left[\left(\mathrm{M}^{+}+1\right)-\right.$ 2CO], 383 and $382\left(\mathrm{M}^{+}-3 \mathrm{CO}\right), 327$ and $329\left[\mathrm{M}^{+}-\mathrm{Fe}(\mathrm{CO})_{3}\right]$.

[4-(p-Bromophenyl)-1-(1,3-dithiolan-2-ylidene)-3Ebuten-2-one $\mathrm{Fe}_{2}(\mathrm{CO})_{5}$, (4c). Purification: CC, Hexane/ AcOEt, 9:1. Deep red solid (18\%), m.p.:99 ${ }^{\circ} \mathrm{C}$ (dec). ${ }^{1} \mathrm{H}$ NMR (300 MHz, $\left.\mathrm{C}_{6} \mathrm{D}_{6}\right): \delta 1.48\left(\mathrm{~m}, 2 \mathrm{H}, \mathrm{FeSCH}_{2}\right), 2.26$ $\left(\mathrm{m}, 2 \mathrm{H}, \mathrm{SCH}_{2}\right), 6.92(\mathrm{~s}, 1 \mathrm{H}, \mathrm{CH}=\mathrm{CSFe}), 6.67$ and 7.01

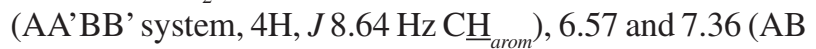
system, $2 \mathrm{H}, J 16.08 \mathrm{~Hz}, \mathrm{CHC} \underline{\mathrm{HCO}}) .{ }^{13} \mathrm{C} \mathrm{NMR}(75 \mathrm{MHz}$, $\left.\mathrm{C}_{6} \mathrm{D}_{6}\right): \delta 30.5\left(\mathrm{FeSCH}_{2}\right), 35.6\left(\mathrm{SCH}_{2}\right), 122.8\left(\mathrm{C}_{i} \mathrm{Br}\right), 124.5$ (ㅁCSFe), 128.8 ( $\left.\underline{\mathrm{CH}}_{o} \mathrm{C}_{i} \mathrm{CH}\right), 129.4$ (CHCHCO), 131.7

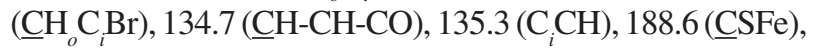
189.0 (ㅁ), 211.6 and 213.5 (M-CO). IR (KBr) $v_{\max } / \mathrm{cm}^{-1}$ : 2059, 2017 (M-CO); 1641 (CO). M.S.-FAB ${ }^{+}(\mathrm{m} / \mathrm{z}): 579$ and $580\left[\mathrm{M}^{+}+1\right], 552\left(\mathrm{M}^{+}-\mathrm{CO}\right), 524\left(\mathrm{M}^{+}-2 \mathrm{CO}\right), 495$ and 497 $\left[\left(\mathrm{M}^{+}+1\right)-3 \mathrm{CO}\right], 440$ and $438\left[\mathrm{M}^{+}-\mathrm{Fe}(\mathrm{CO})_{3}\right]$.

$\eta^{4}$-[4-(p-Methoxiphenyl)-1-(1,3-dithiolan-2-ylidene)3E-buten-2-one $\mathrm{Fe}(\mathrm{CO})_{3}$, (5b). Purification: CC, Hexane/ AcOEt, 96:4. Red solid (20\%), m.p.:176 ${ }^{\circ} \mathrm{C}$ (dec.). ${ }^{1} \mathrm{H}$ NMR $\left(300 \mathrm{MHz}, \mathrm{C}_{6} \mathrm{D}_{6}\right): \delta 2.40\left(\mathrm{~m}, 4 \mathrm{H}, \mathrm{CH}_{2}\right), 3.20\left(\mathrm{~s}, 3 \mathrm{H}, \mathrm{CH}_{3} \mathrm{O}\right)$, 3.59 (d, 1H, J 8.94 Hz, CHC틍), 5.44 (d, 1H, J 8.94 Hz,

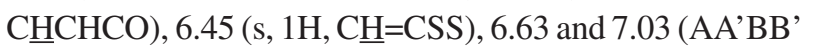
system, $\left.4 \mathrm{H}, J 8.26 \mathrm{~Hz} \mathrm{C}_{\text {arom }}\right) .{ }^{13} \mathrm{C} \mathrm{NMR}\left(75 \mathrm{MHz}, \mathrm{CDCl}_{3}\right)$ : $\delta 35.3$ and $38.8\left(\mathrm{~S}_{\mathrm{CH}_{2}}\right), 54.5\left(\mathrm{CH}_{3} \mathrm{O}\right), 62.4$ ( $\left.\mathrm{CH} \underline{\mathrm{CHCO}}\right)$,

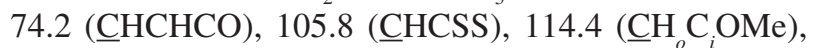
$128.5\left(\underline{\mathrm{CH}}_{o} \mathrm{C} C \mathrm{CH}\right), 131.3\left(\underline{\mathrm{C}}_{i} \mathrm{CH}\right), 141.4\left(\underline{\mathrm{C}}_{i} \mathrm{OMe}\right), 154.0$ (ㄷS), 158.9 (ㅁ), 214.1 (M-CO). IR (KBr) $v_{\max } / \mathrm{cm}^{-1}: 2056$, 1998 and 1979 (M-CO), 1543 (CO). M.S.-FAB ${ }^{+}$ $(\mathrm{m} / \mathrm{z}): 419\left[\mathrm{M}^{+}+1\right], 391\left[\left(\mathrm{M}^{+}+1\right)-\mathrm{CO}\right], 363\left[\left(\mathrm{M}^{+}+1\right)-2 \mathrm{CO}\right]$, $334\left(\mathrm{M}^{+}-3 \mathrm{CO}\right), 279\left(\mathrm{M}^{+}-\mathrm{Fe}(\mathrm{CO})_{3}\right)$.

[4-(p-Methoxiphenyl)-1-(1,3-dithiolan-2-ylidene)-3Ebuten-2-one $\mathrm{Fe}_{2}(\mathrm{CO})_{5}$, (5c). Purification: CC, Hexane/ AcOEt, 9:1. Deep red solid (28\%), m.p.:164-168 ${ }^{\circ} \mathrm{C}$ (dec.). RMN ${ }^{1} \mathrm{H}\left(300 \mathrm{MHz}, \mathrm{C}_{6} \mathrm{D}_{6}\right): \delta 1.54$ and $2.39(\mathrm{~m}, 2 \mathrm{H}$, $\mathrm{FeSCH}_{2}$ ), 3.18(s, 3H, CH $\left.\mathrm{O}\right), 3.22$ and 4.27 (m, 2H, $\mathrm{SCH}_{2}$ ), 6.58 and 7.13 (AA'BB' system, $4 \mathrm{H}, J 8.79 \mathrm{~Hz} \mathrm{C}_{\text {arom }}$ ), 6.77

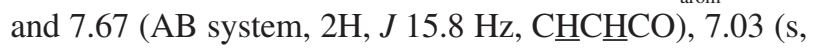
$1 \mathrm{H}, \mathrm{C} \underline{\mathrm{H}}=\mathrm{CSFe}) .{ }^{13} \mathrm{CNMR}\left(75 \mathrm{MHz}, \mathrm{C}_{6} \mathrm{D}_{6}\right): \delta 30.0\left(\mathrm{FeSCH}_{2}\right)$,
$35.3\left(\mathrm{SCH}_{2}\right), 54.6\left(\mathrm{CH}_{3} \mathrm{O}\right), 114.2$ ( $\left.\underline{\mathrm{CH}}_{o} \mathrm{C}_{i} \mathrm{OMe}\right), 120.8$ ( $\underline{\mathrm{CH}}-$ CSFe), 124.0 (CHCHCO), 129.0 ( $\left.\underline{\mathrm{CH}} \mathrm{C}_{i} \mathrm{CH}\right), 129.7$ (C $\left.{ }_{i} \mathrm{CH}\right)$,

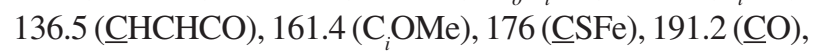
219.5 (M-CO). IR (KBr) $v_{\max } / \mathrm{cm}^{-1}: 2060,2020$ (M-CO), 1603 (CO). M.S.-FAB $(\mathrm{m} / \mathrm{z}): 531\left[\mathrm{M}^{+}+1\right], 474\left(\mathrm{M}^{+}-2 \mathrm{CO}\right)$, $446\left(\mathrm{M}^{+}-3 \mathrm{CO}\right) ; 419$ [(M+1)- 4CO].

$\eta^{4}, \kappa^{N}-[4-[p-(N, N$-diethylamino $)$-phenyl]-1-(1,3dithiolan-2-ylidene)-3E-buten-2-one $\mathrm{Fe}_{2}(\mathrm{CO})_{7},(\boldsymbol{6 b})$. Purification: CC, Hexane/AcOEt, 95:5. Deep red oil, (7\%). ${ }^{1} \mathrm{H} \mathrm{NMR}\left(300 \mathrm{MHz}, \mathrm{CDCl}_{3}\right.$ ): $\delta 0.89\left(\mathrm{q}, 4 \mathrm{H}, \mathrm{CH}_{2} \mathrm{~N}\right), 1.23$ (t, $\left.6 \mathrm{H}, \underline{\mathrm{C}}_{3} \mathrm{CH}_{2} \mathrm{~N}\right), 3.43$ (m, 4H, $\left.\mathrm{CH}_{2} \mathrm{~S}\right), 6.7$ (s, 1H, CHCSS), 5.34 (AB system, $2 \mathrm{H}, \mathrm{CH}=\mathrm{CH}$ ), 6.66 and 7.32 (AA'BB' system, $\left.4 \mathrm{H}, J 8.1 \mathrm{~Hz}, \mathrm{CH}_{\text {arom }}\right)$. IR $\left(\mathrm{CHCl}_{3}\right) v_{\text {máx }} / \mathrm{cm}^{-1}: 2067$, 2072, 2016 and 2003, (M-CO). M.S. $\left(\mathrm{FAB}^{+}\right) \mathrm{m} / 2: 599\left(\mathrm{M}^{+}-\right.$ $\mathrm{CO}), 571\left(\mathrm{M}^{+}-2 \mathrm{CO}\right), 543\left(\mathrm{M}^{+}-3 \mathrm{CO}\right), 431\left[\mathrm{M}^{+}-\mathrm{Fe}(\mathrm{CO})_{5}\right], 403$ $\left[\mathrm{M}^{+}-\mathrm{Fe}(\mathrm{CO})_{6}\right]$.

[4-[p-(N,N-diethylamino)-phenyl]-1-(1,3-dithiolan-2ylidene)-3E-buten-2-one $\mathrm{Fe}_{2}(\mathrm{CO})_{5},(\boldsymbol{6} \mathrm{c})$. Purification: $\mathrm{CC}$, Hexane/AcOEt, 92:8. Deep red oil, (25\%). ${ }^{1} \mathrm{H}$ NMR (300 $\left.\mathrm{MHz}, \mathrm{CDCl}_{3}\right): \delta 1.26\left(\mathrm{t}, 6 \mathrm{H}, \mathrm{CH}_{3} \mathrm{CH}_{2} \mathrm{~N}\right), 3.37\left(\mathrm{q}, 4 \mathrm{H}, \mathrm{CH}_{2} \mathrm{~N}\right)$, 3.16 and $1.53\left(2 \mathrm{~m}, 4 \mathrm{H}, \mathrm{CH}_{2} \mathrm{~S}\right), 7.08$ (s, 1H, CHCSFe), 6.69 and $7.22(\mathrm{~s}, 2 \mathrm{H}, \mathrm{CH}=\mathrm{CH}), 6.61$ and 7.37 (AA'BB' system, $\left.4 \mathrm{H}, J 8.6 \mathrm{~Hz}, \mathrm{CH}_{\text {arom }}\right) .{ }^{13} \mathrm{C} \mathrm{NMR}\left(75 \mathrm{MHz}, \mathrm{CDCl}_{3}\right): \delta 12.7$ $\left(\mathrm{CH}_{3} \mathrm{CH}_{2} \mathrm{~N}\right), 31.0\left(\mathrm{CH}_{2} \mathrm{SFe}\right), 36.1\left(\mathrm{CH}_{2} \mathrm{~S}\right), 44.5\left(\mathrm{CH}_{2} \mathrm{~N}\right)$,

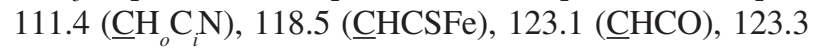
$\left(\underline{\mathrm{C}}_{i} \mathrm{CH}\right), 129.3\left(\underline{\mathrm{CH}}_{o} \mathrm{C} \mathrm{CH}\right), 136.9(\mathrm{Ph} \underline{\mathrm{CH}}), 148.6\left(\underline{\mathrm{C}}_{i} \mathrm{~N}\right), 188.1$ (CSFe), $191.5(\mathrm{CO}), 207.0(\mathrm{CO}-\mathrm{M})$. IR $\left(\mathrm{CHCl}_{3}\right) v_{\text {máx }} / \mathrm{cm}^{-1}$ : 2003, 2016 (M-CO), 1632 (C=O) M.S. $\left(\mathrm{FAB}^{+}\right) \mathrm{m} / z: 572$ $\left(\mathrm{M}^{+}+1\right), 543\left(\mathrm{M}^{+}-\mathrm{CO}\right), 515\left(\mathrm{M}^{+}-2 \mathrm{CO}\right), 487\left(\mathrm{M}^{+}-3 \mathrm{CO}\right), 431$ $\left[\mathrm{M}^{+}-(\mathrm{CO})_{5}\right], 403\left[\mathrm{M}^{+}-\mathrm{Fe}(\mathrm{CO})_{4}\right]$.

4-(p-aminophenyl)-1-(1,3-dithiolan-2-ilydene)-3Ebuten-2-one, (8) Purification: CC, Hexane/AcOEt, 6:4. Orange solid (75\%), m.p.: 233-235 ${ }^{\circ} \mathrm{C} ;{ }^{1} \mathrm{H}$ NMR (300 MHz, $\left.\mathrm{CDCl}_{3}\right): \delta 3.38\left(\mathrm{~m}, 4 \mathrm{H}, \mathrm{CH}_{2} \mathrm{~S}\right), 6.57\left(\mathrm{~s}, 2 \mathrm{H}, \mathrm{NH}_{2}\right), 6.82(\mathrm{~s}$, 1H, C트SS), 6.59 and 7.54 (AB system, $2 \mathrm{H}, J 15.81 \mathrm{~Hz}$, $\mathrm{CH}=\mathrm{CH}$ ), 6.63 and 7.37 (AA'BB' system, 4H, J 8.4 Hz, $\left.\mathrm{CH}_{\text {arom }}\right) .{ }^{13} \mathrm{C} \mathrm{NMR}\left(75 \mathrm{MHz}, \mathrm{CDCl}_{3}\right): \delta 35.3\left(\mathrm{CH}_{2} \mathrm{~S}\right), 40.1$ $\left(\mathrm{CH}_{2} \mathrm{~S}\right), 112.3$ (ㄷCSS), $114.9\left(\underline{\mathrm{CH}}_{o} \mathrm{C}_{i} \mathrm{NH}_{2}\right), 123.6$ (ㅁHCO), $125.4\left(\underline{\mathrm{C}}_{i} \mathrm{CH}\right), 130.1\left(\underline{\mathrm{CH}}_{o} \mathrm{C} \mathrm{CH}\right), 142.4(\mathrm{Ph} \underline{\mathrm{CH}}), 147.5$ $\left(\underline{\mathrm{C}}_{i} \mathrm{NH}_{2}\right), 168.4(\mathrm{CSS}), 184.5(\mathrm{CO})$. IR $\left(\mathrm{CHCl}_{3}\right) v_{\text {máx }} / \mathrm{cm}^{-1}$ : $3408(\mathrm{~N}-\mathrm{H}), 1620(\mathrm{C}=\mathrm{O}), 1498(\mathrm{C}=\mathrm{C})$. M.S.-E.I. $(70 \mathrm{eV})$ $m / z$ (\%): 263[M+] (100), 235(40), 146(25).

$\kappa^{N-}$ [4-(p-Aminophenyl)-1-(1,3-dithiolan-2-ylidene)-3Ebuten-2-one $\mathrm{Fe}(\mathrm{CO})_{4}$, (9). Purification: CC, Hexane/ AcOEt, 9:1. Brown solid, (2\%), m.p.: $166^{\circ} \mathrm{C}$ (dec). IR $\left(\mathrm{CHCl}_{3}\right) v_{\text {máx }} / \mathrm{cm}^{-1}: 3409(\mathrm{~N}-\mathrm{H}), 2061$ and $2019(\mathrm{CO}-\mathrm{M})$, $1600(\mathrm{C}=\mathrm{O}), 1498(\mathrm{C}=\mathrm{C})$. M.S.-E.I. $(70 \mathrm{eV}) \mathrm{m} / z(\%)$ : 431[ $\left.\mathrm{M}^{+}\right](15), 403(10)\left(\mathrm{M}^{+}-\mathrm{CO}\right), 375(5)\left(\mathrm{M}^{+}-2 \mathrm{CO}\right), 347(3)$ $\left(\mathrm{M}^{+}-3 \mathrm{CO}\right), 263(100)\left[\left(\mathrm{M}^{+}-\mathrm{Fe}(\mathrm{CO})_{4}\right]\right.$.

$\kappa^{N}-\{4-[p-(N, N$-diethylamino $)$-phenyl]-1-(1,3-dithiolan2-ylidene)-3E-buten-2-one $\} \mathrm{Fe}(\mathrm{CO})_{4}$, (10). Purification: 
CC, Hexane: AcOEt, 99:1. Red solid, (2\%) m.p.:98-101 ${ }^{\circ} \mathrm{C}$ (dec). ${ }^{1} \mathrm{H}$ NMR (300 MHz, $\left.\mathrm{CDCl}_{3}\right): \delta 0.86\left(\mathrm{q}, 4 \mathrm{H}, \mathrm{CH}_{2} \mathrm{~N}\right)$, $1.17\left(\mathrm{t}, 6 \mathrm{H}, \mathrm{CH}_{3} \mathrm{CH}_{2} \mathrm{~N}\right), 3.37\left(\mathrm{~m}, 4 \mathrm{H}, \mathrm{CH}_{2} \mathrm{~S}\right), 6.82(\mathrm{~s}, 1 \mathrm{H}$, CHCSS), 6.56 and 7.57 (AB system, 2H, J $15.66 \mathrm{~Hz}$, $\mathrm{CH}=\mathrm{CH}$ ), 6.62 and 7.39 (AA'BB' system, 4H, $J 8.1 \mathrm{~Hz}$, $\left.\mathrm{CH}_{\text {arom }}\right)$. IR $\left(\mathrm{CHCl}_{3}\right) v_{\text {máx }} / \mathrm{cm}^{-1}: 2003,2016$ (M-CO) 1632(C=O). E.M. $\left(\mathrm{FAB}^{+}\right) \mathrm{m} / \mathrm{z}:$ 487( $\left.\mathrm{M}^{+}-28\right), 431\left(\mathrm{M}^{+}-2 \mathrm{CO}\right)$, 403( $\left.\mathrm{M}^{+}-3 \mathrm{CO}\right), 319\left[\mathrm{M}^{+}-\left(\mathrm{Fe}(\mathrm{CO})_{4}\right]\right.$.

$X$-ray crystal structure determinations of compounds $\mathbf{3}$ and $\mathbf{4 b}$

Data collection and refinement parameters are summarized in Table 3. The diffraction data for $\mathbf{3}$ and $\mathbf{4 b}$ were collected on a Bruker Smart Apex CCD diffractometer with $\mathrm{MoK} \alpha$ radiation, $\lambda=0.71063 \AA$. Each data set was corrected for Lorentz and polarization effects and analytical absorption corrections based on face indexed were applied. The structures were solved by direct methods ${ }^{18}$ and each structure was refined by full-matrix least squares on $F^{2}$ using all data with the all non-hydrogen atoms assigned anisotropic displacement parameters and hydrogen atoms bound to carbon atoms inserted at calculated position with isotropic temperature factor 1.2 times the Uiso of the parent carbon atom. The program used in the final refinements was SHELXL-97. ${ }^{19}$ Selected bond lengths and bond angles are shown in Tables 1-2.

\section{Supplementary Information}

Crystallographic data (excluding structure factors) for the structures in this paper have been deposited with the Cambridge Crystallographic Data Centre as supplementary publication no CCDC No. 251031 for ligand 3 and No. 251032 for complex $\mathbf{4 b}$. Copies of the data can be obtained, free of charge via www.ccdc.cam.ac.uk/conts/retrieving.html (or from the Cambridge Crystallographic Data Centre, CCDC, 12 Union Road, Cambridge CB2 1EZ, UK ; fax: +44 1223336033 ; or e-mail: deposit@ccdc.cam.ac.uk).

\section{Acknowledgements}

We thank DGAPA for financial support (Project \# IN242004). The technical assistance of Rocio Patiño, Luis Velasco and Javier Pérez is gratefully acknowledged.

\section{References}

1. Hegedus L.S.; Transition Metals in the Synthesis of Complex Organic Molecules, University Science Books: CA, 1994, pp. 131-142, 220-225.
2. Howell, J.A. S.; Johnson, B.f. G.; Josty, P.L.; Lewis, J.; J. Organomet. Chem. 1972, 39, 329; Domingos, J. P.; Howell, J. S. A.; Johnson, B. F. G.; Lewis, J.; Inorg. Synth. 1976, 16, 103; Fleckner, H.; Grevels, F.W.; Hess, D.; J. Am. Chem. Soc. 1984, 106, 2027; Knölker, H. J.; Baum, G.; Foitzik, N.; Goesmann, H.; Gonser, P.; Jones, P. G.; Röttele, H.; Eur. J. Inorg. Chem. 1998, 993.

3. Brandsna, L.; Vasilesky, S. F.; Verbre, H. D.; Application of Transition Metal Catalyst in Organic Chemistry, Springer: New York, 1999, p.254-307; Barton D. H. R.; Gunatilaka, A. A. L.; Nakanishi, T.; Patin, H.; Widdowson, D. A.; Worth, B. R.; J. Chem Soc. Perkin Trans.1 1976, 821; Evans, G.; Johnson, B. F.G.; Lewis, J.; J.Organomet. Chem. 1975, 102, 507.

4. Cox, L. R.; Ley, S. V.; Chem Soc. Rev. 1998, 27, 301; Takemoto, Y.; Baba, Y.; Yoshikawa, N.; Iwata, C.; Tanaka, T.; Ibuka, T.; Chem. Commun. 1998, 1911; Knölker, H. J.; Chem. Soc. Rev. 1999, 28, 151; Frank-Neuman, M.; Olson, P. J.; Synlett 1991, 891; Donaldson, W. A.; Bell, P. T.; Wang, Z.; Bennett, D. W.; Tetrahedron Lett. 1994, 32, 5892; Prahald, V.; Donaldson, W. A.; Tetrahedron Lett. 1996, 37, 9169; Benvegnu, T. J.; Toupet, L. J.; Grée, R.; Tetrahedron 1996, 52, 11811; Benvegnu, T. J.; Grée, R.; Tetrahedron 1996, 52, 11821. Iwata, C.; Takemoto, Y.; Chem. Commun. 1996, 2497.

5. Alvarez, C.; Gutiérrez, R.; Toscano, R. A.; Moya, M.; Velasco, L.; Rosas, R.; Tapia, R.; Penieres, G.; J. Coord. Chem. 1999, 48, 383; Alvarez-Toledano, C.; Gutiérrez-Pérez, R.; Toscano, R. A.; Moya-Cabrera, M.; Haquet, T.; Ortega, M. C.; Cabrera, A.; Polyhedron 2001, 20, 215; Alvarez-Toledano, C.; Enríquez, J.; Toscano, R. A.; Martínez-García, M.; Cortés-Cortés, E.; Osornio, Y.; García, O.; Gutierrez-Pérez, R.; J. Organomet. Chem. 1999, 577, 38; Ortega, C.; Gutiérrez, R.; Sharma, P.; Gómez, E.; Toscano, A.; López, J. G.; Penieres, G.; Alvarez C.; Trends Organomet. Chem. 2002, 4, 19.

6. Ortega-Jiménez, F.; Ortega-Alfaro, M. C.; López-Cortés, J. G.; Gutiérrez-Pérez, R.; Toscano, R. A.; Velasco-Ibarra, L.; Peña-Cabrera, E.; Alvarez-Toledano, C.; Organometallics 2000, 19, 4127.

7. Karl Dieter, R.; Tetrahedron 1986, 42, 3029; Yates, P.; Lynch, T. R.; Moore, D. R.; Can. J. Chem. 1971, 49, 1467.

8. Shriver, D. F.; Whitmire K. H. In Comprehensive Organometallic Chemistry. The Synthesis, Reactions and Structures of Organometallic Compounds; Wilkinson, G.; Stone, F. G.; Abel, E. W., eds.; Pergamon Press: Oxford, 1982,vol. 4, p.251,252.

9. Huges, D. L.; Leigh, G. L.; Paulson, D.; Inorg. Chim. Acta 1986, 120, 191; King, R. B.; J. Am. Chem. Soc. 1963, 85, 1584.

10. Bernès, S.; Toscano, R. A.; Cano, A. C.; Mellado, O. G.; Alvarez-Toledano, C.; Rudler, H.; Daran, J.; J. Organomet. Chem. 1995, 498, 15; Alvarez-Toledano, C.; HernándezOrtega, S.; Bernès, S.; Gutiérrez-Pérez, R.; García-Mellado, O.; J. Organomet. Chem. 1997, 549, 49. 
11. Ortega-Alfaro, M. C.; Hernández, N.; Cerna, I.; López-Cortés, J. G.; Gómez, E.; Toscano, R. A.; Alvarez-Toledano, C.; J. Organomet. Chem. 2004, 689, 885.

12. Howell, J. A. S.; Johnson, B. F.; Josty, P. L.; Lewis, J.; J. Organomet. Chem. 1972, 39, 329.

13. Geribaldi, S.; Azzaro, M.; Spectrochim. Acta 1982, 38A, 779; Ewing, D. F. In Correlation Analysis in Chemistry: Recent Advances; Chapman, N. B.; Shorter, J., eds.; Plenum: NY, 1978.

14. Hansch, C.; Leo, A.; Taft, R. W.; Chem. Rev. 1991, 91, 165.

15. Smith, M. B.; March, J.; Advanced Organic Chemistry. Reactions, Mechanims and Structure. $5^{\text {th }}$ ed., Willey: USA, 2001, p 363-380.
16. Pearson, A.J.; Iron Compounds in Organic Chemistry, Academic Press: CA, 1994, p. 199-270.

17. Reichenbach, G.; Cardaci, G.; Aloisi, G. G.; J. Organomet. Chem. 1977, 134, 47.

18. Altomare, A.; Cascarano, G.; Giacovazzo, C.; Guagliardi, A.; Burla, M. C.; Polidori, G.; Canalli, M.; J. Appl. Cryst. 1994, $27,435$.

19. Sheldrick, G. M.; SHELXL-97 Program for Refinement of Crystal Structures, Univ. of Goettingen, Germany, 1997.

Received: October 13, 2004 Published on the web: March 15, 2005 